\title{
Time-domain asymptotic- numerical solution for transient scattered electric field by a coated conducting cylinder covered with a thin lossy dielectric material
}

\author{
Keiji Goto $^{\text {a) }}$, Ryo Asai, Naokatsu Sumikawa, \\ and Taweedej Santikul \\ Department of Communication Engineering, National Defense Academy, \\ 1-10-20 Hashirimizu, Yokosuka, Kanagawa 239-8686, Japan \\ a) keigoto@nda.ac.jp
}

\begin{abstract}
We derive a time-domain (TD) asymptotic-numerical solution for the transient scattered electric field by a coated conducting cylinder covered with a thin lossy dielectric material by substituting the frequencydomain asymptotic solution into the transient scattered electric field integral. The TD asymptotic-numerical solution is represented by a combination of each transient scattered field element which is obtained from the numerical integration. The validity and computational efficiency of the TD asymptoticnumerical solution are confirmed by comparing with the reference solution. We show that, since the TD asymptotic-numerical solution can extract and observe each pulse wave element from the response waveform even when the pulse wave elements overlap mutually, it is effective understanding the scattering phenomena in detail.
\end{abstract}

Keywords: time-domain, asymptotic-numerical solution, transient scattered field, coated conducting cylinder, thin lossy dielectric material

Classification: Electromagnetic theory

\section{References}

[1] H. T. Kim and N. Wang: IEEE Trans. Antennas Propag. 37 (1989) 1463. DOI: $10.1109 / 8.43566$

[2] T. B. A. Senior and J. L. Volakis, ed.: Approximate Boundary Conditions in Electromagnetics (IEE, London, 1995) chap. 7.

[3] P. E. Hussar: IEEE Trans. Antennas Propag. 46 (1998) 998. DOI:10.1109/8. 704801

[4] T. Ida, T. Ishihara and K. Goto: IEICE Trans. Electron. E88-C (2005) 2124. DOI:10.1093/ietele/e88-c.11.2124

[5] K. Goto and L. H. Loc: IEICE Electron. Express 10 (2013) 20130100. DOI:10. 1587/elex.10.20130100 
[7] K. Goto, T. Santikul, R. Asai and N. Sumikawa: The Papers of Technical Meeting on Electromagnetic Theory, IEE Japan, EMT-14-152 (2014) 23.

[8] L. B. Felsen and N. Marcuvitz, ed.: Radiation and Scattering of Waves (IEEE Press (Classic Reissue), New Jersey, 1994) sec. 6.7.

[9] P. H. Pathak: Radio Sci. 14 (1979) 419. DOI:10.1029/RS014i003p00419

[10] M. Abramowitz and I. A. Stegun, ed.: Handbook of Mathematical Functions (Dover Publications, Inc., New York, 1972).

[11] K. Goto, T. Ishihara and L. B. Felsen: IEEE Trans. Antennas Propag. 50 (2002) 1109. DOI:10.1109/TAP.2002.803471

[12] K. Goto, M. Sawada, K. Mori and Y. Horii: IEICE Electron. Express 11 (2014) 20130963. DOI:10.1587/elex.11.20130963

\section{Introduction}

The studies in the high-frequency (HF) scattering analysis by an impedance or a coated cylinder have been important research subjects in the application area such as the low-observability techniques for the radar cross section of an aircraft fuselage $[1,2,3,4]$.

We have recently derived the frequency-domain (FD) extended UTD (uniform geometrical theory of diffraction) and the FD modified UTD solution for the scattered field by a coated conducting cylinder covered with a thin lossy dielectric material $[5,6,7]$. The extended UTD and modified UTD solutions can connect uniformly the asymptotic solution in the deep lit region and that in the deep shadow region through the transition region (TR) near the shadow boundary (SB). We have also derived the FD asymptotic solutions for the direct geometric optical ray (DGO) and the reflected geometric optical ray (RGO) effective in the deep lit region [6, 7].

In this paper, by extending the FD asymptotic solution in [5, 7], we derive a time-domain (TD) asymptotic-numerical solution for the transient scattered electric field when a HF modulated pulse wave is incident on a coated conducting cylinder covered with a lossy dielectric material. The thickness of the dielectric material is thin as compared with the wavelength of a central angular frequency of a pulse source function. The TD asymptotic-numerical solution is represented by a combination of the transient scattered electric field elements which consist of the DGO, the RGO, the extended UTD for the pseudo surface diffracted ray (pseudo SD), the modified UTD for the SD series, and the lowest order SD.

The validity and computational efficiency of the TD asymptotic-numerical solution are confirmed by comparing with the reference solution. We show that, since the TD asymptotic-numerical solution can extract and observe each pulse wave element from the response waveform even when the pulse wave elements overlap mutually, it is effective understanding the scattering phenomena in detail. The time convention $\exp (-i \omega t)$ is adopted and suppressed in this paper.

\section{Formulation and frequency-domain (FD) asymptotic solution}

Figs. 1(a) and 1(b) show a coated conducting cylinder with radius $a$ covered with a homogeneous medium $2\left(\varepsilon_{2}^{*}, \mu_{0}\right)$ of thickness $t(=a-b)$ and the coordinate systems $(x, y, z)$ and $(\rho, \phi)$. Notation $\varepsilon_{2}^{*}$ denotes a complex dielectric constant of 


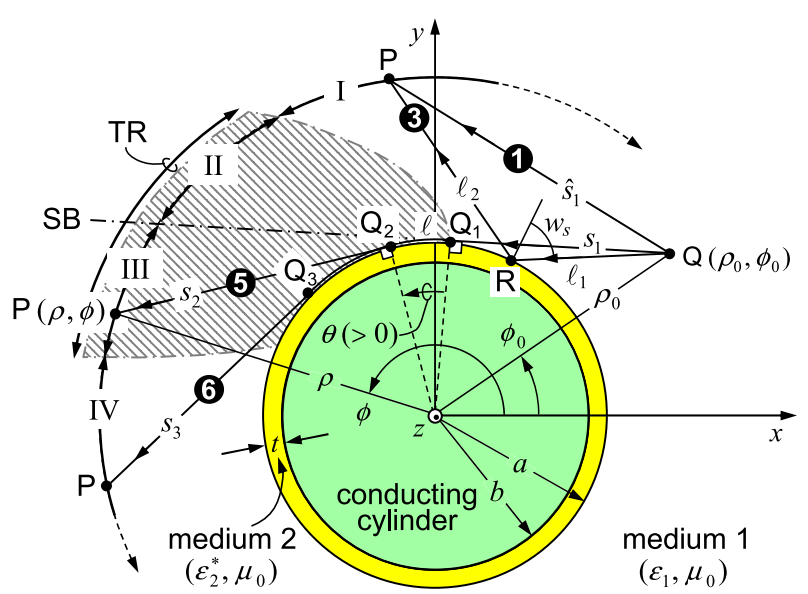

(a)

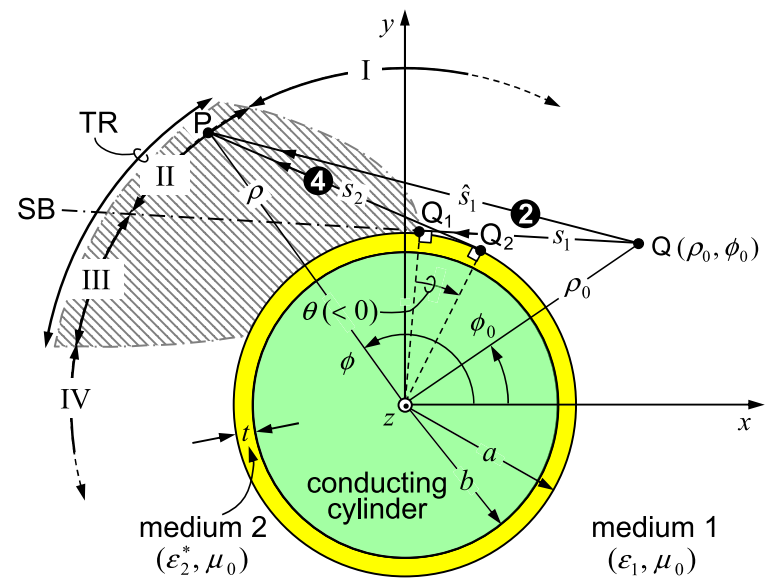

(b)

Fig. 1. Scattering phenomena by a coated conducting cylinder covered with a thin lossy dielectric material and coordinate systems $(x, y, z)$ and $(\rho, \phi) . \mathrm{Q}\left(\rho_{0}, \phi_{0}\right)$ : source point, $\mathrm{P}(\rho, \phi)$ : observation point, SB: shadow boundary, and TR: transition region. Scattered field elements: DGO (1) and (2), RGO (3), pseudo SD (4), and SD (5 and (6). (a) Propagation paths of scattered field elements observed in the regions I, III, and IV. (b) Propagation paths of scattered field elements observed in the region II.
() IEICE 2015

DOI: $10.1587 /$ elex.12.20141110 Received November 25, 2014 Accepted December 4, 2014

Publicized January 7, 2015

Copyedited February 10, 2015 
TR near the SB. The regions III and IV represent a shadow side of the TR and a deep shadow region far away from the SB, respectively. We have also shown in Figs. 1(a) and 1(b) the propagation paths of the DGO (() and (2), the RGO (3), the pseudo SD (4), and the SD (5 and (6).

The asymptotic analysis method for the FD scattered electric field when the HF electric type cylindrical wave is incident on the coated cylinder has been discussed in $[5,7]$ in detail. In the following sections, only the results needed in the TD asymptotic-numerical analysis for the transient scattered electric field in Section 3 is summarized.

\subsection{FD asymptotic solution for DGO}

The FD asymptotic solution $E_{z, \mathrm{DGO}}(\omega)$ for the DGO effective in the regions I and II (see 1 in Fig. 1(a) and 2 in Fig. 1(b)) along the path $(\mathrm{Q} \rightarrow \mathrm{P})$ from the source point $\mathrm{Q}$ to the observation point $\mathrm{P}(\rho, \phi)$ is given by $[7,8]$

$$
E_{z, \mathrm{DGO}}(\omega)=\frac{i}{4} \sqrt{\frac{2}{\pi k_{1} \hat{s}_{1}}} \exp \left(i k_{1} \hat{s}_{1}-i \pi / 4\right), \quad \hat{s}_{1}=\overline{\mathrm{QP}} .
$$

In order to simplify notation of $E_{z, \mathrm{DGO}}(\omega)$ in (1), the wavenumber $k_{1}$ which is a function of $\omega$ is used.

\subsection{FD asymptotic solution for RGO}

The FD asymptotic solution $E_{z, \mathrm{RGO}}(\omega)$ for the RGO effective in the region I with the path $\mathrm{Q} \rightarrow \mathrm{R} \rightarrow \mathrm{P}$ (see 3 in Fig. 1(a)) which arrives at the point $\mathrm{P}$ after radiating from the point $\mathrm{Q}$ and reflecting at the point $\mathrm{R}$ is expressed by $[1,7,9]$

$$
E_{z, \mathrm{RGO}}(\omega)=E_{z, \mathrm{in}}(\mathrm{R}) \Gamma\left(w_{s}\right) \sqrt{\frac{\tilde{\rho}^{\gamma}}{\tilde{\rho}^{\gamma}+\ell_{2}}} \exp \left(i k_{1} \ell_{2}\right), \quad \ell_{2}=\overline{\mathrm{RP}} .
$$

Here $E_{z, \text { in }}(\mathrm{R})$ and $\Gamma\left(w_{s}\right)$ are the incident cylindrical wave propagating from the point $\mathrm{Q}$ to the reflection point $\mathrm{R}$ and the reflection coefficient at the point $\mathrm{R}$ and are defined as follows [7].

$$
\begin{gathered}
E_{z, \text { in }}(\mathrm{R})=\frac{i}{4} \sqrt{\frac{2}{\pi k_{1} \ell_{1}}} \exp \left(i k_{1} \ell_{1}-i \pi / 4\right), \quad \ell_{1}=\overline{\mathrm{QR}}, \\
\Gamma\left(w_{s}\right)=\frac{\cos w_{s}-Y\left(v_{s}\right)}{\cos w_{s}+Y\left(v_{s}\right)}, \quad v_{s}=k_{1} a \sin w_{s}, \\
Y(v) \sim i \frac{Z_{1}}{Z_{2}} \cdot \frac{\sqrt{\left(k_{2}^{*} a\right)^{2}-v^{2}}}{k_{2}^{*} a} \cot \left(B_{1, v}-B_{2, v}\right), \quad Z_{1}=\sqrt{\frac{\mu_{0}}{\varepsilon_{1}}}, \quad Z_{2}=\sqrt{\frac{\mu_{0}}{\varepsilon_{2}}} \\
B_{1, v}=\sqrt{\left(k_{2}^{*} a\right)^{2}-v^{2}}-v \cos ^{-1} \frac{v}{k_{2}^{*} a}, \quad B_{2, v}=\sqrt{\left(k_{2}^{*} b\right)^{2}-v^{2}}-v \cos ^{-1} \frac{v}{k_{2}^{*} b} .
\end{gathered}
$$

Where $w_{s}$ denotes the incident angle of the RGO (see Fig. 1(a)) and $Y(v)$ a normalized admittance $[1,5,7]$, and $Z_{1}\left(Z_{2}\right)$ the characteristic impedance in the medium 1 (in the medium 2).

The square root term $\sqrt{\tilde{\rho}^{\gamma} /\left(\tilde{\rho}^{\gamma}+\ell_{2}\right)}$ in (2) denotes the divergence factor and $\tilde{\rho}^{\gamma}$ is the caustic distance of RGO $[1,7,9]$. 


\subsection{FD extended UTD solution for pseudo SD}

The FD extended UTD solution $E_{z \text {,extended UTD }}(\omega)$ for the pseudo SD effective in the region II with the path $\mathrm{Q} \rightarrow \mathrm{Q}_{1} \curvearrowright \mathrm{Q}_{2} \rightarrow \mathrm{P}$ (see 4 in Fig. 1(b)) which retreats to the surface diffraction point $\mathrm{Q}_{2}$ by the surface diffraction after emanating from the point $\mathrm{Q}$ and entering into the surface diffraction point $\mathrm{Q}_{1}$, carries out the surface diffraction again at the point $\mathrm{Q}_{2}$, and then arrives at the point $\mathrm{P}$, is given by [7]

$$
E_{z, \text { extended UTD }}(\omega)=E_{z, \text { in }}\left(\mathrm{Q}_{1}\right) \tilde{T}\left(\mathrm{Q}_{1}, \mathrm{Q}_{2}\right) \frac{\exp \left(i k_{1} s_{2}\right)}{\sqrt{s_{2}}} .
$$

Here $E_{z, \text { in }}\left(\mathrm{Q}_{1}\right)$ denotes the incident cylindrical wave propagating from the point $\mathrm{Q}$ to the point $\mathrm{Q}_{1}$ and $\tilde{T}\left(\mathrm{Q}_{1}, \mathrm{Q}_{2}\right)$ is the transmission function, which expresses the scattering phenomena along the $\operatorname{arc} \mathrm{Q}_{1} \curvearrowright \mathrm{Q}_{2}$ of the coating surface $(\rho=a)$ from the point $\mathrm{Q}_{1}$ to the point $\mathrm{Q}_{2}$, and are defined by

$$
\begin{gathered}
E_{z, \text { in }}\left(\mathrm{Q}_{1}\right)=\frac{i}{4} \sqrt{\frac{2}{\pi k_{1} s_{1}}} \exp \left(i k_{1} s_{1}-i \pi / 4\right), \quad s_{1}=\overline{\mathrm{QQ}_{1}}, \\
\tilde{T}\left(\mathrm{Q}_{1}, \mathrm{Q}_{2}\right)=-M \sqrt{\frac{2}{k_{1}}} \exp \left(i k_{1} \tilde{\ell}\right)\left[-\frac{\exp (i \pi / 4)}{2 \sqrt{\pi} \tilde{\xi}} F(\tilde{X})+p_{s}^{e}\left(\tilde{\xi}, L, q_{c}(\tau)\right)\right] .
\end{gathered}
$$

In (9), the distance $\tilde{\ell}$ of the creeping wave (CW), the Fresnel function $F(\tilde{X})$, and the extended Pekeris function $p_{s}^{e}\left(\tilde{\xi}, L, q_{c}(\tau)\right)$ are defined as follows.

$$
\begin{gathered}
\tilde{\ell}=-a|\theta|, \quad \theta=\left|\phi-\phi_{0}\right|-\cos ^{-1} \frac{a}{\rho_{0}}-\cos ^{-1} \frac{a}{\rho} \\
F(\tilde{X})=-i 2 \tilde{X} \exp \left(-i \tilde{X}^{2}\right) \int_{\tilde{X}}^{\infty} \exp \left(i \tau^{2}\right) d \tau, \quad \tilde{X}=\sqrt{2 k_{1} L} \frac{|\theta|}{2} \\
p_{s}^{e}\left(\tilde{\xi}, L, q_{c}(\tau)\right)=\frac{e^{-i \pi / 4}}{2 \sqrt{\pi}} \int_{i \infty}^{0} \frac{w_{2}^{\prime}(\tau)-q_{c}(\tau) w_{2}(\tau)}{w_{1}^{\prime}(\tau)-q_{c}(\tau) w_{1}(\tau)} \exp \left[i \tilde{\xi} \tau+i \frac{M^{2}}{2 k_{1} L} \tau^{2}\right] d \tau \\
+\frac{e^{-i \pi / 4}}{\sqrt{\pi}} \int_{0}^{\infty} \frac{A i^{\prime}(\tau)-q_{c}(\tau) A i(\tau)}{w_{1}^{\prime}(\tau)-q_{c}(\tau) w_{1}(\tau)} \exp \left[i \tilde{\xi} \tau+i \frac{M^{2}}{2 k_{1} L} \tau^{2}\right] d \tau, \\
w_{1}(\tau)=A i(\tau) \mp i B i(\tau), \quad w_{1}^{\prime}(\tau)=A i^{\prime}(\tau) \mp i B i^{\prime}(\tau), \\
q_{c}(\tau)=i M Y(\tau), \quad \tilde{\xi}=-M|\theta|, \quad M=\left(\frac{k_{1} a}{2}\right)^{1 / 3}, \quad L=\frac{s_{1} s_{2}}{s_{1}+s_{2}}, \quad s_{2}=\overline{\mathrm{Q}_{2} \mathrm{P}},
\end{gathered}
$$

where $A i(\tau)$ and $B i(\tau)$ are the Airy functions [10] and the primes (') on these functions denote the derivative with respect to the argument. Notation $Y(\tau)$ in (14) represents a function obtained by $v=k_{1} a+M \tau, M=\left(k_{1} a / 2\right)^{1 / 3}$, replacing $v$ by $\tau$ in (5) associated with (6). The last term in (7) represents a cylindrical wave propagating along the path $\mathrm{Q}_{2} \rightarrow \mathrm{P}$ from the point $\mathrm{Q}_{2}$ to the point $\mathrm{P}$.

\subsection{FD modified UTD solution for SD series}

The FD modified UTD solution $E_{z \text {,modified } \operatorname{UTD}(M)}(\omega)$ for the SD series effective in the regions III and IV with the path $\mathrm{Q} \rightarrow \mathrm{Q}_{1} \curvearrowright \mathrm{Q}_{2} \rightarrow \mathrm{P}$ (see $\mathbf{5}$ in Fig. 1(a)) which propagates along the arc $\mathrm{Q}_{1} \curvearrowright \mathrm{Q}_{2}$ to the point $\mathrm{Q}_{2}$ by the surface diffraction after the cylindrical wave excited at the point $\mathrm{Q}$ enters into the point $\mathrm{Q}_{1}$, carries out the surface diffraction at the point $\mathrm{Q}_{2}$ after that, and arrives at the point $\mathrm{P}$, is expressed by $[5,7]$ 


$$
\begin{gathered}
E_{z, \text { modified } \operatorname{UTD}(M)}(\omega)=E_{z, \text { in }}\left(\mathrm{Q}_{1}\right) \sum_{m=1}^{M}\left[D_{m}\left(\mathrm{Q}_{1}\right) A_{m}\left(\mathrm{Q}_{1}\right) \exp \left(i k_{1} \ell-\Omega_{m} \ell\right)\right. \\
\left.\cdot D_{m}\left(\mathrm{Q}_{2}\right) A_{m}\left(\mathrm{Q}_{2}\right)\right] \frac{\exp \left(i k_{1} s_{2}\right)}{\sqrt{s_{2}}}, \quad s_{2}=\overline{\mathrm{Q}_{2} \mathrm{P}}, \\
\ell=a \theta, \quad \theta=\left|\phi-\phi_{0}\right|-\cos ^{-1} \frac{a}{\rho_{0}}-\cos ^{-1} \frac{a}{\rho} .
\end{gathered}
$$

Here $E_{z, \text { in }}\left(\mathrm{Q}_{1}\right)$ denotes the incident wave in (8) and $D_{m}\left(\mathrm{Q}_{1}\right) A_{m}\left(\mathrm{Q}_{1}\right)$ $\left(D_{m}\left(\mathrm{Q}_{2}\right) A_{m}\left(\mathrm{Q}_{2}\right)\right)$ the new surface diffraction coefficient at the point $\mathrm{Q}_{1}\left(\mathrm{Q}_{2}\right)[5$, 7]. The conventional GTD diffraction coefficient $D_{m}\left(\mathrm{Q}_{1,2}\right)$, the modification coefficient $A_{m}\left(\mathrm{Q}_{1,2}\right)$, and the attenuation coefficient $\Omega_{m}$ are defined by $[4,5,7]$. Notation $M$ is the truncation number of the SD series solution.

When the point $\mathrm{P}$ moves to the region IV from the region III, the FD asymptotic solution in (15) may be represented by only the lowest order SD with $M=1$ along the path $\mathrm{Q} \rightarrow \mathrm{Q}_{1} \curvearrowright \mathrm{Q}_{3} \rightarrow \mathrm{P}$ (see 6 in Fig. 1(a)) [11].

$$
\begin{aligned}
E_{z, \mathrm{SD}}(\omega) & \sim E_{z, \text { modified } \operatorname{UTD}(M=1)}(\omega) \\
& =E_{z, \text { in }}\left(\mathrm{Q}_{1}\right)\left[D_{1}\left(\mathrm{Q}_{1}\right) A_{1}\left(\mathrm{Q}_{1}\right) e^{i k_{1} \ell-\Omega_{1} \ell} D_{1}\left(\mathrm{Q}_{3}\right) A_{1}\left(\mathrm{Q}_{3}\right)\right] \frac{\exp \left(i k_{1} s_{3}\right)}{\sqrt{s_{3}}},
\end{aligned}
$$

here $\ell\left(=\mathrm{Q}_{1} \curvearrowright \mathrm{Q}_{3}\right)$ denotes the distance of the $\mathrm{CW}$ from the point $\mathrm{Q}_{1}$ to the point $\mathrm{Q}_{3}$ and $s_{3}\left(=\overline{\mathrm{Q}_{3} \mathrm{P}}\right)$ the distance from the point $\mathrm{Q}_{3}$ to the point $\mathrm{P}$.

\subsection{FD asymptotic solution for scattered electric field}

The FD asymptotic solution $E_{z, a s y}(\omega)$ for the scattered electric field $E_{z}(\omega)$ arriving at the point $\mathrm{P}$ from the counterclockwise direction without encircling the coated cylinder after radiated from the point $\mathrm{Q}$ can be represented by a combination of each FD asymptotic solution summarized in the foregoing sections.

$$
E_{z}(\omega) \sim E_{z, a s y}(\omega)=\sum_{i=\mathrm{I}}^{\mathrm{IV}} U_{i}(\mathrm{P}) \sum_{r} E_{z, r}(\omega),
$$

where $r=\mathrm{DGO}$, RGO, extended UTD, modified $\mathrm{UTD}(M)$, SD.

Where $E_{z, \mathrm{DGO}}(\omega), E_{z, \mathrm{RGO}}(\omega), E_{z, \text { extended } \mathrm{UTD}}(\omega), E_{z, \operatorname{modified~} \mathrm{UTD}(M)}(\omega)$, and $E_{z, \mathrm{SD}}(\omega)$ denote respectively the DGO in (1), the RGO in (2), the extended UTD in (7), the modified UTD in (15), and the SD in (17). The function $U_{i}(\mathrm{P}), i=\mathrm{I}$, II, III, IV, is defined as $U_{i}(\mathrm{P})=1$ (or $U_{i}(\mathrm{P})=0$ ) when the observation point $\mathrm{P}$ is (is not) located in the region $i$.

\section{Time-domain (TD) asymptotic-numerical solution}

\subsection{Transient scattered electric field integral}

The transient scattered electric field $y(t)$ by a coated cylinder covered with a thin lossy dielectric material can be expressed by the following inverse Fourier transform of the product of the FD scattered electric filed $E_{z}(\omega)$ and the frequency spectrum $S(\omega)$ of a pulse source function $s(t)$ [12].

$$
y(t)=\frac{1}{2 \pi} \int_{-\infty}^{\infty} E_{\mathrm{z}}(\omega) S(\omega) \exp (-i \omega t) d \omega
$$


We assume the following HF modulated pulse source $s(t)$ defined by the product of the modulated wave $s_{0}(t)$ and the carrier wave $\exp \left[-i \omega_{0}\left(t-t_{0}\right)\right]$ whose central angular frequency is $\omega_{0}$ :

$$
s(t)=\left\{\begin{array}{cl}
s_{0}(t) \exp \left[-i \omega_{0}\left(t-t_{0}\right)\right] & \text { for } 0 \leq t \leq 2 t_{0} \\
0 & \text { for } t<0, t>2 t_{0}
\end{array},\right.
$$

where $t_{0}$ is the constant parameter. Denoting the Fourier transform of $s_{0}(t)$ by $S_{0}(\omega)$, the Fourier transform $S(\omega)$ of $s(t)$ can be represented by

$$
S(\omega)=S_{0}\left(\omega-\omega_{0}\right) \exp \left(i \omega_{0} t_{0}\right) .
$$

After substituting the exact solution calculated from the eigenfunction expansion $[1,2]$ for the FD scattered electric field $E_{z}(\omega)$ and the frequency spectrum $S(\omega)$ in (21) into the integral $y(t)$ in (19), by carrying out the numerical integration of $y(t)$, one may calculate the transient scattered electric field numerically.

In this paper, the numerical solution which can be obtained by applying the fast Fourier transform (FFT) numerical code to the transient scattered electric field integral $y(t)$ in (19) is used as the reference solution in Section 4. The response waveform of $y(t)$ is obtained from the real part of $y(t)$ i.e., $\operatorname{Re}[y(t)]$.

\subsection{TD asymptotic-numerical solution}

Substituting the FD asymptotic solution $E_{z, a s y}(\omega)$ in (18) into (19), one may derive the following representation.

$$
y(t) \sim y_{\text {asy }}(t)=\sum_{i=\mathrm{I}}^{\mathrm{IV}} U_{i}(\mathrm{P}) \sum_{r} y_{r}(t),
$$

where $r=\mathrm{DGO}$, RGO, extended UTD, modified $\operatorname{UTD}(M)$, SD.

In (22), $y_{r}(t)$ denotes the transient scattered electric field element and is represented by the inverse Fourier transform of the product of the FD asymptotic solution $E_{z, r}(\omega)$ (see (18)) and the Frequency spectrum $S(\omega)$ in (21).

$$
y_{r}(t)=\frac{1}{2 \pi} \int_{-\infty}^{\infty} E_{z, r}(\omega) S(\omega) \exp (-i \omega t) d \omega .
$$

The integral $y_{r}(t)$ in (23) is numerically calculable by applying the FFT numerical code. Therefore, we can derive the TD asymptotic-numerical solution for the transient scattered field by substituting the numerical solution $y_{r}(t)$ in (23) into (22).

Since the TD asymptotic-numerical solution $y_{\text {asy }}(t)$ in (22) can extract and observe each pulse wave element $\operatorname{Re}\left[y_{r}(t)\right]$ in (23) from the response waveform $\operatorname{Re}\left[y_{\text {asy }}(t)\right]$ even when the pulse wave elements overlap mutually, it is effective understanding the scattering phenomena by a coated conducting cylinder covered with a thin lossy dielectric material in detail.

\section{Numerical results and discussions}

In this section, we perform the numerical calculations required to assess the validity and computational efficiency of the TD asymptotic-numerical solution derived in Section 3.2 and to interpret the scattering phenomena when the HF modulated 
pulse wave is incident on a coated conducting cylinder covered with a thin lossy dielectric material.

Figs. 2(a) through 2(d) show the response waveform vs. time curves observed in the region I (Fig. 2(a)), the region II (Fig. 2(b)), the region III (Fig. 2(c)), and the region IV (Fig. 2(d)) (see Figs. 1(a) and 1(b)). Numerical parameters used in the calculations are given in the caption of Fig. 2 and the time $t$ is set $t=0$ when the HF modulated pulse wave is radiated from the source point Q. In this case, the SB (see Figs. 1(a) and 1(b)) is located at $\left|\phi-\phi_{0}\right|=95.7^{\circ}$ and the range of the region II is defined by $84.8^{\circ} \leq\left|\phi-\phi_{0}\right| \leq \mathrm{SB}$ as a case where the variable of $\tilde{X}$ in (11) satisfies $0 \leq \tilde{X} \leq \sqrt{3}$. In the same manner, the range of the region III is defined by $\mathrm{SB} \leq\left|\phi-\phi_{0}\right| \leq 106.6^{\circ}$ as a case where the following variable $X$ satisfies $0 \leq X \leq \sqrt{3}$ (see (8) in [5] or (13) in [7]).

$$
X=\sqrt{2 k_{1} L} \frac{\theta}{2}, \quad \theta=\left|\phi-\phi_{0}\right|-\cos ^{-1} \frac{a}{\rho_{0}}-\cos ^{-1} \frac{a}{\rho} .
$$

The response waveform of the reference solution $\operatorname{Re}[y(t)]$ (-- -: red dashed curve) has been calculated by applying the FD exact solution and the FFT numerical code to (19). The response waveform of the TD asymptotic-numerical solution $\operatorname{Re}\left[y_{a s y}(t)\right]$ ( - : solid curve) has been obtained from (22) by applying the FFT numerical code to $y_{r}(t)$ in (23) (see (25), (26), (27), and (28)). It is confirmed in Figs. 2(a) to 2(d) that each TD asymptotic-numerical solution (-) agrees excellently with the reference solution (---).

In the following, we discuss the interpretation method of the response waveforms in Fig. 2(a) through Fig. 2(d) and show the computation times of the reference and the TD asymptotic-numerical solution.

As shown in Fig. 2(a), two wave packets are observed in the response waveform. The region I in Fig. 1(a) is shown the DGO (() ) and the RGO (3) arriving at the point P. From the arrival time difference of the two wave packets, the $\operatorname{Re}[y(t)]$ in (19) can be divided into the pulse wave elements $(1$ and (3. While, when set the function $U_{i}(\mathrm{P})$ in $(22)$ to $U_{\mathrm{I}}(\mathrm{P})=1$ and $U_{\mathrm{II}}(\mathrm{P})=U_{\mathrm{III}}(\mathrm{P})=U_{\mathrm{IV}}(\mathrm{P})=0$, the $\operatorname{Re}\left[y_{a s y}(t)\right]$ is simplified and is given by

$$
\operatorname{Re}\left[y_{\text {asy }}(t)\right]=\operatorname{Re}\left[y_{\mathrm{DGO}}(t)\right]+\operatorname{Re}\left[y_{\mathrm{RGO}}(t)\right] \text { for region } \mathrm{I} .
$$

Since the $\operatorname{Re}\left[y_{a s y}(t)\right]$ in (25) is represented by the sum of the pulse wave elements $\operatorname{Re}\left[y_{\mathrm{DGO}}(t)\right]$ and $\operatorname{Re}\left[y_{\mathrm{RGO}}(t)\right]$, it can be recognized immediately that the first wave packet is the DGO ((1) and the second one is the RGO (3). As to a computation time, it takes 64 seconds for the $\operatorname{Re}[y(t)]$ and approximately 1 second for the $\operatorname{Re}\left[y_{\text {asy }}(t)\right]$.

Fig. 2(b) is depicted one wave packet in the response waveform. As shown in Fig. 1(b), the region II is arrived at the DGO (2) and the pseudo SD (4). In this case, the reference solution $\operatorname{Re}[y(t)]$ cannot decompose the response waveform into the two pulse waves 2 and (4. Whereas setting $U_{i}(\mathrm{P})$ in $(22)$ to $U_{\mathrm{II}}(\mathrm{P})=1$ and $U_{\mathrm{I}}(\mathrm{P})=U_{\mathrm{III}}(\mathrm{P})=U_{\mathrm{IV}}(\mathrm{P})=0$, the $\operatorname{Re}\left[y_{\text {asy }}(t)\right]$ is expressed by

$$
\operatorname{Re}\left[y_{\text {asy }}(t)\right]=\operatorname{Re}\left[y_{\mathrm{DGO}}(t)\right]+\operatorname{Re}\left[y_{\text {extended UTD }}(t)\right] \text { for region II. }
$$

In order to interpret the wave packet in Fig. 2(b) in detail, Fig. 3 shows two pulse wave elements $\operatorname{Re}\left[y_{\mathrm{DGO}}(t)\right]$ (Fig. 3(a)) and $\operatorname{Re}\left[y_{\text {extended }} \mathrm{UTD}(t)\right]$ (Fig. 3(b)), 


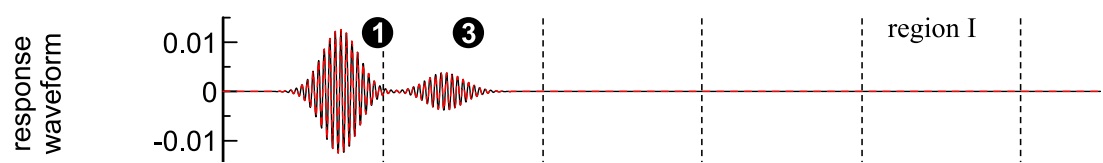

(a)

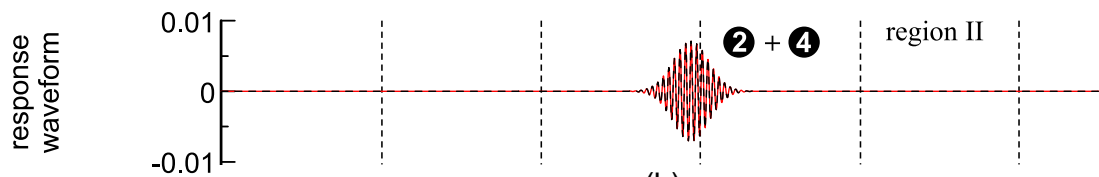

(b)

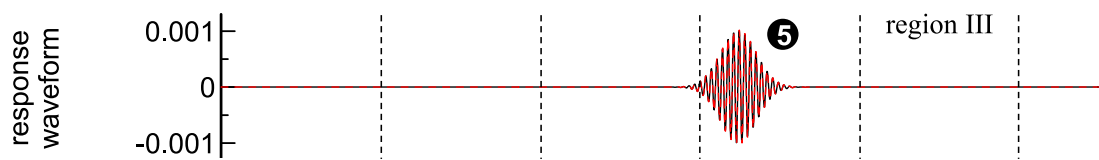

(c)

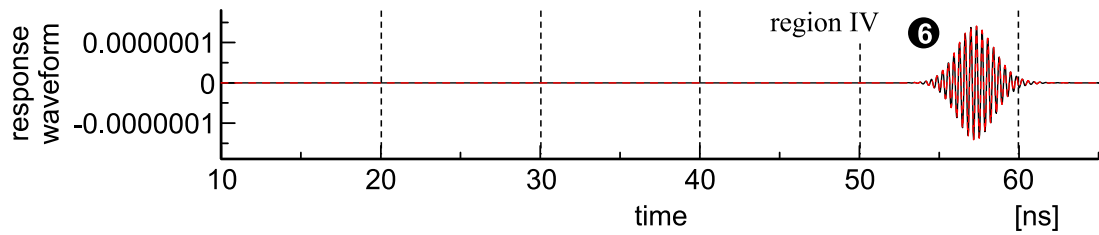

(d)

Fig. 2. Response waveforms scattered by the coated conducting cylinder covered with a thin lossy dielectric material and observed in the region I (Fig. 2(a)), the region II (Fig. 2(b)), the region III (Fig. 2(c)), and the region IV (Fig. 2(d)). Numerical parameters: $a=5 \mathrm{~m}, \varepsilon_{1}=\varepsilon_{0}, \varepsilon_{2}^{*}=\varepsilon_{0}\left(\varepsilon_{2 r}+i \sigma /\left(\omega \varepsilon_{0}\right)\right), \quad \varepsilon_{2 r}=$ $5 \varepsilon_{0}, \quad \sigma=0.159 \mathrm{~S} / \mathrm{m}, \quad t=0.4 \lambda_{0}\left(\lambda_{0}=2 \pi c_{1} / \omega_{0}\right)$, source point $\mathrm{Q}\left(\rho_{0}, \phi_{0}\right)=\left(1.4 a, 0^{\circ}\right)$. Pulse source $s(t)$ in (20) with $s_{0}(t)=$ $\exp \left[-\left(t-t_{0}\right)^{2} /\left(4 d^{2}\right)\right]$ used in the calculation $\left(t_{0}=4 \times 10^{-9} \mathrm{~s}\right.$, $\left.d=8 \times 10^{-10} \mathrm{~s}, \quad \omega_{0}=1.8 \times 10^{10} \mathrm{rad} / \mathrm{s}\right)$. Observation point $\mathrm{P}(\rho, \phi)=(1.6 a, \phi) ; \phi=30^{\circ}$ (Fig. 2(a)), $\phi=90^{\circ}$ (Fig. 2(b)), $\phi=100^{\circ}$ (Fig. 2(c)), and $\phi=150^{\circ}$ (Fig. 2(d)). - - -: reference solution in (19), —: TD asymptotic-numerical solution in (22) associated with (23).

and the response waveform $\operatorname{Re}\left[y_{a s y}(t)\right]$ (Fig. 3(c)) which are provided by (26). Since the $\operatorname{Re}\left[y_{\text {asy }}(t)\right]$ in Fig. 3(c) agrees excellently with the $\operatorname{Re}[y(t)]$ in Fig. 2(b), the wave packet in Fig. 2(b) can be interpreted as a bunch of the pulse waves by superposition of the DGO (2) and the SD (4). The computation times of the $\operatorname{Re}[y(t)]$ and the $\operatorname{Re}\left[y_{a s y}(t)\right]$ are 66 seconds and 19 seconds, respectively.

As shown in Fig. 2(c), one wave packet is observed in the response waveform. By referring to the region III in Fig. 1(a), the reference solution $\operatorname{Re}[y(t)]$ can be checked with the SD $(\boldsymbol{5})$. On the other hand, setting the $U_{i}(\mathrm{P})$ and the truncation number $M$ in $(22)$ to $U_{\text {III }}(\mathrm{P})=1, U_{\mathrm{I}}(\mathrm{P})=U_{\mathrm{II}}(\mathrm{P})=U_{\mathrm{IV}}(\mathrm{P})=0$, and $M=10$, the $\operatorname{Re}\left[y_{\text {asy }}(t)\right]$ is given by

$$
\operatorname{Re}\left[y_{\text {asy }}(t)\right]=\operatorname{Re}\left[y_{\text {modified }} \operatorname{UTD}(M=10)(t)\right] \text { for region III. }
$$

From (27), the wave packet of Fig. 2(c) can be interpreted as a bunch of 10 pulse wave elements. The computation times of the $\operatorname{Re}[y(t)]$ and the $\operatorname{Re}\left[y_{\text {asy }}(t)\right]$ are respectively 67 seconds and 20 seconds.

Finally, one wave packet is observed in the response waveform in Fig. 2(d). By referring to the region IV in Fig. $1(\mathrm{a})$, the $\operatorname{Re}[y(t)]$ can be checked with the SD 


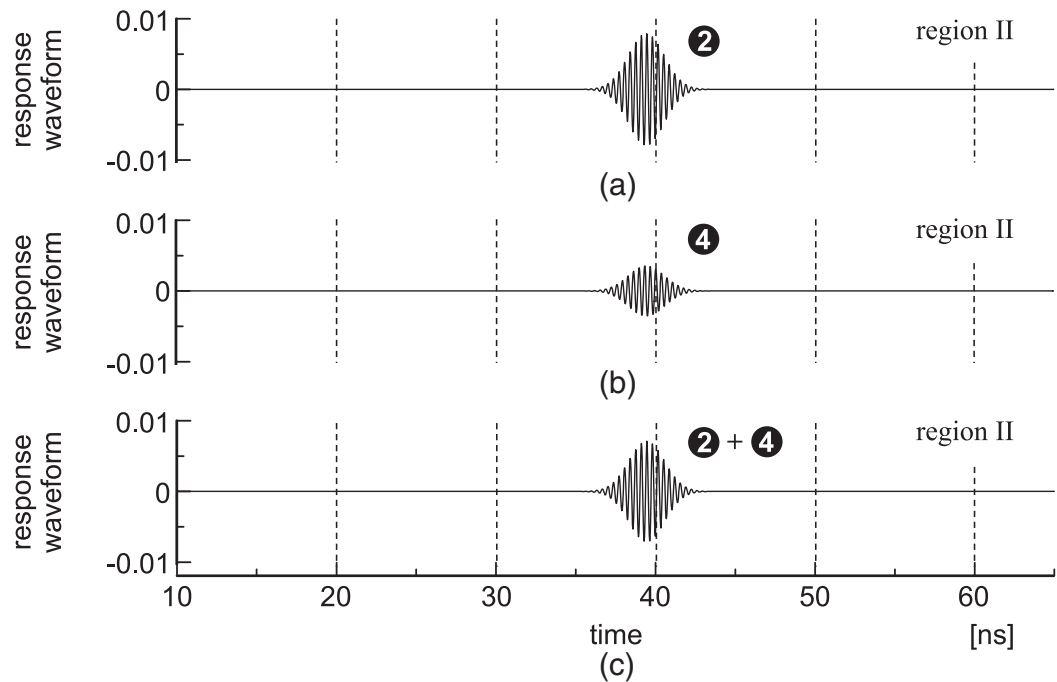

Fig. 3. Pulse wave elements $\operatorname{Re}\left[y_{\mathrm{DGO}}(t)\right] \quad$ (Fig. 3(a)) and $\operatorname{Re}\left[y_{\text {extended UTD }}(t)\right]$ (Fig. 3(b)) and response waveform $\operatorname{Re}\left[y_{a s y}(t)\right]$ (Fig. 3(c)) observed in the region II. Numerical parameters used in the calculations in Figs. 3(a), 3(b), and 3(c) are the same as those used in Fig. 2(b).

(6). In the same manner, setting the $U_{i}(\mathrm{P})$ in $(22)$ to $U_{\mathrm{IV}}(\mathrm{P})=1$ and $U_{\mathrm{I}}(\mathrm{P})=$ $U_{\text {II }}(\mathrm{P})=U_{\text {III }}(\mathrm{P})=0$, the $\operatorname{Re}\left[y_{\text {asy }}(t)\right]$ is represented by

$$
\operatorname{Re}\left[y_{a s y}(t)\right]=\operatorname{Re}\left[y_{\mathrm{SD}}(t)\right] \text { for region IV. }
$$

It can be recognized from (28) that the wave packet in Fig. 2(d) is the SD (6). The computation time is 76 seconds for the $\operatorname{Re}[y(t)]$ and 4 seconds for the $\operatorname{Re}\left[y_{a s y}(t)\right]$.

From the above-mentioned discussions, the TD asymptotic-numerical solution in (22) associated with (23) has been able to extract and observe each pulse wave element from the response waveform even when the pulse wave elements overlap mutually. The computational efficiency of the TD asymptotic-numerical solution was demonstrated as compared with the computation time of the reference solution. Hence, it was confirmed that the TD asymptotic-numerical solution is effective understanding the scattering phenomena by a coated conducting cylinder covered with a thin lossy dielectric material in detail.

\section{Conclusion}

We have derived the time-domain (TD) asymptotic-numerical solution for the transient scattered electric field by a coated conducting cylinder covered with a thin lossy dielectric material. By comparing with the reference solution, we confirmed the validity and computational efficiency of the TD asymptotic-numerical solution. We showed that the TD asymptotic-numerical solution proposed here is effective understanding the scattering phenomena in detail.

\section{Acknowledgement}

This work was supported in part by the Grant-in-Aid for Scientific Research (C) 